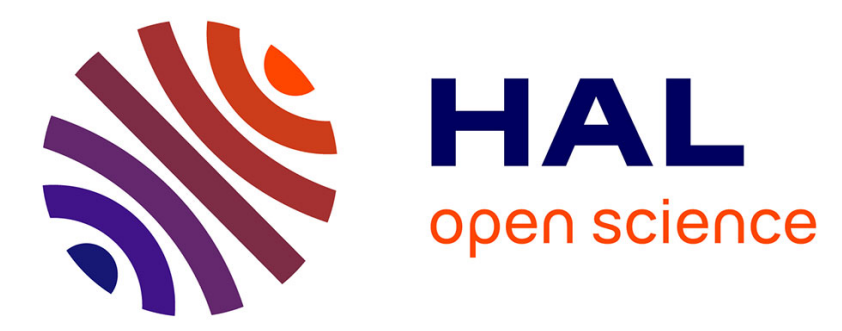

\title{
Energy equation for certain approximate models of long-wave hydrodynamics
}

\author{
Zinaida Fedotova, Gayaz Khakimzyanov, Denys Dutykh
}

\section{To cite this version:}

Zinaida Fedotova, Gayaz Khakimzyanov, Denys Dutykh. Energy equation for certain approximate models of long-wave hydrodynamics. Russian Journal of Numerical Analysis and Mathematical Modelling, 2014, 29 (3), pp.167-178. 10.1515/rnam-2014-0013 . hal-00998927

\section{HAL Id: hal-00998927 \\ https://hal.science/hal-00998927}

Submitted on 7 Jan 2019

HAL is a multi-disciplinary open access archive for the deposit and dissemination of scientific research documents, whether they are published or not. The documents may come from teaching and research institutions in France or abroad, or from public or private research centers.
L'archive ouverte pluridisciplinaire HAL, est destinée au dépôt et à la diffusion de documents scientifiques de niveau recherche, publiés ou non, émanant des établissements d'enseignement et de recherche français ou étrangers, des laboratoires publics ou privés.

\section{(1)(1) $\$(0)$}

Distributed under a Creative Commons Attribution - NonCommercial - ShareAlikel 4.0 


\title{
Zinaida I. Fedotova, Gayaz S. Khakimzyanov*, and Denys Dutykh Energy equation for certain approximate models of long-wave hydrodynamics
}

\begin{abstract}
A new derivation of completely nonlinear weakly-dispersive shallow water equations is given without assumption of flow potentiality. Boussinesq type equations are derived for weakly nonlinear waves above a moving bottom. It is established that the total energy balance condition holds for all nonlinear dispersion models obtained here.
\end{abstract}

Keywords: Conservative laws, ideal incompressible fluid, nonlinear dispersion equations, surface waves.

MSC 2010: 76B15

Zinaida I. Fedotova, Gayaz S. Khakimzyanov: Institute of Computational Technologies, Siberian Branch of the Russian Academy of Sciences, Novosibirsk 630090, Russia. E-mail: khak@ict.nsc.ru

Denys Dutykh: Université de Savoie, Campus Scientifique, 73376 Le Bourget-du-Lac, France

The most common way to obtain long-wave approximations of hydrodynamic equations for an incompressible fluid is the expansion of basic hydrodynamic parameters over powers of the vertical coordinate, or over powers of the long-wave parameter determined by the ratio of the average depth of water area to the typical wavelength. These methods are very formal. The systems of nonlinear-dispersive (NLD) equations constructed with the use of those methods were justified in particular cases (e.g. theorems on convergence, correctness, and stability $[2,14,15])$. However, a wide class of NLD models is widely used for solving applied problems on propagation and interaction of long surface waves. This contributes to the growth of computing power of modern computer systems. At the same time, the requirements to the accuracy and robustness of numerical solutions also increase which, in this turn, creates new demands to the quality of approximate hydrodynamic models and the corresponding numerical algorithms.

In the last decade, many papers were focused on improvement of NLD models, their justification, extension to more general cases $[2-5,7,10,11,13]$. Nevertheless, there are still many problems to be solved in this area for today.

An important characteristic of a system of equations describing the fluid dynamics is the energy conservation law $[1,7,9,18,19]$. It is not an independent equation in the case of an ideal homogeneous incompressible fluid, but is obtained as an algebraic corollary of equations of continuity and motion. Passing to approximate models, we may loss important properties of the original hydrodynamic system (such as the invariance of the main group of transformations, or the validity of conservation laws).

The notion of 'energy' was used for approximate hydrodynamic models in many papers $[2,18]$ focused on justification of those models because with the use of a positive function being an analogue of the physical energy one can introduce an appropriate norm in the spaces where the convergence and correctness issues of the Cauchy problem for the corresponding equations are considered.

In this paper, we study the problem of energy variation laws for approximate models of long-wave hydrodynamics taking into account a movable bottom and their relationship with the total energy conservation law of the original 3D model. Since, based on problems of numerical modelling, the form of the energy balance equation for an NLD model has an independent importance relative to the necessity to control the accuracy of numerical algorithms, it is sufficient to apply only the method of equivalent transformation to obtain it from the equation of the existing NLD model. Expanding the range of problems, in particular, related to modification of a completely nonlinear NLD model in order to simplify it, a variational formulation presents an expedient approach [4, 9]. The direct approach to simplify the model by applying the Boussinesq condition well-known in the theory of long waves leads to models not satisfying an analogue of conservation law even in the case of an even bottom (for example, this applies to the well-known Peregrine model [16]). 
The main result of the paper is the derivation of a balance relation for the momentum and total energy for a completely nonlinear NLD model and for models of Boussinesq type. The form of this relation has the same meaning as in the three-dimensional fluid dynamics. This is a positive feature of approximate models, which provides the richness of content for those models and can be used in estimation of the accuracy of numerical algorithms.

The paper has the following structure. In Section 1, we present the formulation of energy conservation law in the 3D hydrodynamic model. In Section 2, we write the law of energy variation for the Green-Naghdi model. Section 3 contains an asymptotic derivation of the NLD model whose determining equations formally coincide with the equations of the Green-Naghdi model after equivalent transformations, but the derivation conditions are less restrictive in this case. The momentum and energy conservation laws are obtained for this model in the form close to that of gas dynamics. Based on obtained equations, in Sections 4 and 5, we derive NLD models with simplified dispersion terms keeping also the important features of original models such as the invariance with respect to the Galilean transformation and the presence of adequate laws of momentum and energy variation.

\section{Euler equations and energy conservation law}

Let us consider the flow of an ideal incompressible fluid in a layer bounded from below by a movable bottom given by some function $z=-h(x, y, t)$ and from above by a free boundary described by a function $z=\eta(x, y, t)$, where $t$ is the time, $x, y, z$ are the coordinates of a point in the Cartesian coordinate system $O x y z$, the axis $O z$ is directed vertically upward, the coordinate plane $O x y$ coincides with the unperturbed free surface. We assume that the acceleration of gravity $g$ and the density $\rho$ of the fluid are constant in the whole layer. In the three-dimensional formulation, we have to calculate the velocity vector $\boldsymbol{U}=(u, v, w)$, the pressure $p$, and the function $\eta$ satisfying the following Euler system of equations:

$$
\begin{aligned}
\nabla \cdot \boldsymbol{u}+w_{z} & =0 \\
\boldsymbol{u}_{t}+(\boldsymbol{u} \cdot \nabla) \boldsymbol{u}+w \boldsymbol{u}_{z}+\nabla p & =0 \\
w_{t}+\boldsymbol{u} \cdot \nabla w+w w_{z}+p_{z} & =-g
\end{aligned}
$$

and the boundary conditions on the bottom and free surface

$$
\left.\left(\eta_{t}+\boldsymbol{u} \cdot \nabla \eta-w\right)\right|_{z=\eta}=0,\left.\quad p\right|_{z=\eta}=0,\left.\quad\left(h_{t}+\boldsymbol{u} \cdot \nabla h+w\right)\right|_{z=-h}=0
$$

where $\nabla=(\partial / \partial x, \partial / \partial y), \boldsymbol{u}=(u, v)$ is the vector of horizontal velocity component, $w$ is the vertical velocity component, $\nabla \cdot \boldsymbol{u}=u_{x}+v_{y}$. In order to simplify the notations in equations (1.1)-(1.3) and in all further formulas including those of transformation to dimensionless variables, we omit the symbol $\rho$ of water density $(\rho \equiv 1)$.

We write the energy equation corresponding to Euler equations (1.1)-(1.3). Multiplying equations (1.2), (1.3) by the velocity components $u, v$, and $w$ and summing the obtained expressions, we get the following equation for the kinetic energy $K=\left(u^{2}+v^{2}+w^{2}\right) / 2$ :

$$
K_{t}+\boldsymbol{u} \cdot \nabla(K+p)+w(K+p)_{z}+g w=0 .
$$

Adding (1.5) to the equation for the potential energy $\Pi=g z$ :

$$
\Pi_{t}+\boldsymbol{u} \cdot \nabla \Pi+w \Pi_{z}-g w=0
$$

we derive the following equation for the total energy $E=K+\Pi$ :

$$
E_{t}+\boldsymbol{u} \cdot \nabla(E+p)+w(E+p)_{z}=0 .
$$

If we add continuity equation (1.1) multiplied by $E+p$ to the latter equation, we obtain the required total energy conservation law in its conservative form:

$$
E_{t}+\nabla \cdot(\boldsymbol{u}(E+p))+(w(E+p))_{z}=0
$$


Equation (1.6) has been derived without taking into account the influence of the boundary. Integrating this equation over the thickness of the fluid layer subject to boundary conditions (1.4), we have

$$
\frac{\partial}{\partial t} \int_{-h}^{\eta} E \mathrm{~d} z+\nabla \cdot \int_{-h}^{\eta} \boldsymbol{u}(E+p) \mathrm{d} z+\left.p\right|_{z=-h} h_{t}=0
$$

This equation describes the variation of the total energy averaged over the fluid layer and takes the form of a conservative conservation law only in the case of a stationary bottom surface. Below, we use it to evaluate the approximation of total energy equations in approximate models describing the propagation of long waves.

The long-wave theory applies expansions of basic hydrodynamic parameters into series over powers of $z+h$ or into series over powers of the small parameter $\mu=h_{0} / L$, where $h_{0}$ and $L$ are the typical depth and wavelength. Substituting such series into equation (1.7) and rejecting the terms of higher orders, we obtain the energy equation for approximate long-wave models. However, we begin with the well-known Green-Naghdi model $[8,12]$ whose energy equation directly follows from (1.7) due to special assumptions on the flow properties.

\section{Energy variation law in the Green-Naghdi model}

The Green-Naghdi model is derived under the assumption that the horizontal velocity component $\boldsymbol{u}$ does not depend on $z$ and the vertical component $w$ is linear with respect to $z$ [8,12]. For $\boldsymbol{u}_{z}=0$ formula (1.7) takes the form

$$
\frac{\partial H \mathcal{E}}{\partial t}+\nabla \cdot\left(\boldsymbol{u} H \mathcal{E}+\boldsymbol{u} \int_{-h}^{\eta} p \mathrm{~d} z\right)+\left.p\right|_{z=-h} h_{t}=0
$$

where $H=\eta+h$ is the total depth and $\mathcal{E}$ denotes the mean energy over depth:

$$
\varepsilon=\frac{1}{H} \int_{-h}^{\eta} E \mathrm{~d} z
$$

The conditions used in the derivation of the Green-Naghdi model imply that the vertical velocity component and the pressure satisfy formulas $[8,10]$ :

$$
\begin{gathered}
w(z)=-D h-(z+h) \nabla \cdot \boldsymbol{u} \\
p(z)=g(H-(z+h))-(H-(z+h)) R_{2}-\left(\frac{H^{2}}{2}-\frac{(z+h)^{2}}{2}\right) R_{1}
\end{gathered}
$$

where

$$
R_{1}=D(\nabla \cdot \boldsymbol{u})-(\nabla \cdot \boldsymbol{u})^{2}, \quad R_{2}=D^{2} h, \quad D=\frac{\partial}{\partial t}+\boldsymbol{u} \cdot \nabla .
$$

By $P$ we denote the pressure averaged over depth of the fluid layer:

$$
P=\frac{1}{H} \int_{-h}^{\eta} p \mathrm{~d} z=g \frac{H}{2}-\frac{H^{2}}{3} R_{1}-\frac{H}{2} R_{2} .
$$

In this case, formula (2.1) can be rewritten in the following form:

$$
\frac{\partial H \mathcal{E}}{\partial t}+\nabla \cdot[\boldsymbol{u} H(\mathcal{E}+\mathcal{P})]+\left.p\right|_{z=-h} h_{t}=0 .
$$

Taking into account (2.2), the total energy takes the form

$$
E=\frac{\boldsymbol{u} \cdot \boldsymbol{u}}{2}+\frac{(D h+(z+h) \nabla \cdot \boldsymbol{u})^{2}}{2}+g z
$$


and for the mean energy $\mathcal{E}$ over depth we have the formula

$$
\mathcal{E}=\frac{\boldsymbol{u} \cdot \boldsymbol{u}}{2}+\frac{H^{2}}{6}(\nabla \cdot \boldsymbol{u})^{2}+\frac{H}{2}(\nabla \cdot \boldsymbol{u}) D h+\frac{(D h)^{2}}{2}+\frac{g(H-2 h)}{2} .
$$

In the case of classic (dispersion-free) shallow water model, the variation of energy has form (2.5) too. However the formulas for the pressure and energy have a simpler form compared to (2.4) and (2.7):

$$
P=\frac{g H}{2}, \quad \mathcal{E}=\frac{\boldsymbol{u} \cdot \boldsymbol{u}}{2}+\frac{g(H-2 h)}{2} .
$$

It is well-known that the energy conservation law plays a fundamental role in justification of the theory of shallow water equations of first approximation [18].

\section{Derivation of the NLD model under a weak restriction on the velocity}

The system of completely nonlinear weakly-dispersive shallow water equations for approximate description of surface waves can be derived under weaker restrictions on the three-dimensional fluid flow velocity than it was done in [8]. Below we present such derivation. It is important to note that the derivation does not use the assumption on the potential flow property, i.e., the flow is assumed to be vortical as was considered in $[8,12]$.

\subsection{Basic assumptions and auxiliary relations}

In order to derive shallow water equations, we introduce typical scales and pass to dimensionless variables. If $L$ is the typical horizontal size, $h_{0}, a_{0}$ are the typical depth and wave amplitude, then we can define dimensionless variables in the following way:

$$
\begin{gathered}
\bar{x}=\frac{x}{L}, \quad \bar{y}=\frac{y}{L}, \quad \bar{z}=\frac{z}{h_{0}}, \quad \bar{t}=\frac{t \sqrt{g h_{0}}}{L}, \quad \bar{h}=\frac{h}{h_{0}}, \quad \bar{\eta}=\frac{\eta}{a_{0}} \\
\overline{\boldsymbol{u}}=\frac{\boldsymbol{u}}{\sqrt{g h_{0}}}, \quad \bar{w}=\frac{L w}{h_{0} \sqrt{g h_{0}}}, \quad \bar{p}=\frac{p}{g h_{0}}, \quad \bar{E}=\frac{E}{g h_{0}} .
\end{gathered}
$$

Formulas (3.2) of dimensionless velocity vector components directly follow from relations (3.1) for the independent variables $x, y, z, t$.

In these new variables, problem (1.1)-(1.4) takes the following form (to simplify the notations, hereafter, we omit the bar over dimensionless variables):

$$
\begin{array}{r}
\nabla \cdot \boldsymbol{u}+w_{z}=0 \\
\boldsymbol{u}_{t}+(\boldsymbol{u} \cdot \nabla) \boldsymbol{u}+w \boldsymbol{u}_{z}+\nabla p=0 \\
\mu^{2}\left(w_{t}+\boldsymbol{u} \cdot \nabla w+w w_{z}\right)+p_{z}+1=0 \\
\left.\left(\alpha \eta_{t}+\alpha \boldsymbol{u} \cdot \nabla \eta-w\right)\right|_{z=\alpha \eta}=0 \\
\left.p\right|_{z=\alpha \eta}=0 \\
\left.\left(h_{t}+\boldsymbol{u} \cdot \nabla h+w\right)\right|_{z=-h}=0
\end{array}
$$

where $\alpha=a_{0} / h_{0}$ is the parameter of nonlinearity, $\mu=h_{0} / L$ is the parameter of dispersion. Below, if not otherwise stated, only dimensionless variables will be used.

The velocity vector in the approximate model is taken as the horizontal velocity component $\boldsymbol{u}$ averaged over depth. Applying to it the transformation to dimensionless form by formulas (3.1), (3.2), we get

$$
\boldsymbol{c}=\left(c_{1}, c_{2}\right)=\frac{1}{H} \int_{-h}^{\alpha \eta} \boldsymbol{u} \mathrm{d} z
$$


where $H=\alpha \eta+h$. Note, that if the velocity vector $\boldsymbol{u}$ does not depend on the vertical coordinate $z$ as in the Green-Naghdi model, then $\boldsymbol{c}$ coincides with $\boldsymbol{u}$.

Integrating continuity equation (3.3) over the thickness of the fluid layer and taking into account boundary conditions (3.6), (3.8) and using formula (3.9), we obtain the following continuity equation for the NLD model [10]:

$$
H_{t}+\nabla \cdot(H \boldsymbol{c})=0 .
$$

In order to derive motion equations for the NLD model, we use the following expansion of the velocity vector into a series over powers of the parameter $\mu^{2}$ :

$$
\boldsymbol{u}=\boldsymbol{u}_{0}+\mu^{2} \boldsymbol{u}_{1}+O\left(\mu^{4}\right), \quad w=w_{0}+\mu^{2} w_{1}+O\left(\mu^{4}\right) .
$$

Since the variation of functions in the direction of the axis $\mathrm{Oz}$ is small in the long-wave approximation in comparison with variations in the horizontal plane, we assume that the principal term $\boldsymbol{u}_{0}$ of the horizontal component $\boldsymbol{u}$ in expansion (3.11) does not depend on the vertical coordinate $z$ :

$$
\left(\boldsymbol{u}_{0}\right)_{z}=0 .
$$

This assumption seems natural for the long-wave hydrodynamic approximation. In particular, one method to obtain the classic dispersion-free shallow water model taking into account only the first term $\boldsymbol{u}_{0}$ in expansion (3.11) (formally, $\mu=0$ ) is based on assumption (3.12).

If we consider well-known derivations of complete NLD models not requiring the potential property of the flow [1,12], then we see the assumption that the horizontal velocity vector $\boldsymbol{u}$ does not depend on $z$ at all. Therefore, restriction (3.12) assumed in this paper without a similar condition on $\boldsymbol{u}_{1}$ is weaker.

Let us stress that, in contrast with well-known papers (e.g., [16]) using expansion (3.11) for derivation of NLD models, in this paper we do not use the potential property of the flow.

Before we derive the motion equations for the velocity $\boldsymbol{c}$, express the functions $\boldsymbol{u}$ and $w$ through $\boldsymbol{c}$ based on expansion (3.11). First, we reveal the relations between $w_{0}$ and $\boldsymbol{u}_{0}$. Substituting the functions $\boldsymbol{u}$ and $w$ of form (3.11) into continuity equation (3.3), we get the relation $\nabla \cdot \boldsymbol{u}_{0}+\left(w_{0}\right)_{z}=0$. Integrating this equality in the vertical coordinate and taking into account that $\boldsymbol{u}_{0}$ does not depend on $z$, we get

$$
(z+h) \nabla \cdot \boldsymbol{u}_{0}+w_{0}(z)-\left.w_{0}(z)\right|_{z=-h}=0 .
$$

Further we use condition (3.8). Taking into account expansion (3.11), it implies

$$
\left.w_{0}\right|_{z=-h}=-D_{0} h, \quad D_{0}=\frac{\partial}{\partial t}+\boldsymbol{u}_{0} \cdot \nabla .
$$

In its turn, this relation and (3.13) imply that the component $w_{0}$ of the vertical velocity component is expressed through $\boldsymbol{u}_{0}$ linearly with respect to $z$ :

$$
w_{0}(z)=-D_{0} h-(z+h) \nabla \cdot \boldsymbol{u}_{0} .
$$

Further, we establish the relations between the velocities $\boldsymbol{c}$ and $\boldsymbol{u}$. Substituting the expansion of the vector $\boldsymbol{u}$ into formula (3.9), we have

$$
\boldsymbol{c}=\frac{1}{H} \int_{-h}^{\alpha \eta}\left(\boldsymbol{u}_{0}+\mu^{2} \boldsymbol{u}_{1}+O\left(\mu^{4}\right)\right) \mathrm{d} z=\boldsymbol{u}_{0}+\mu^{2} \frac{1}{H} \int_{-h}^{\alpha \eta} \boldsymbol{u}_{1} \mathrm{~d} z+O\left(\mu^{4}\right) .
$$

The first formula of (3.11) can be rewritten in the form

$$
\boldsymbol{u}=\boldsymbol{c}+\mu^{2} \boldsymbol{V}+O\left(\mu^{4}\right)
$$

where

$$
\boldsymbol{V}=\boldsymbol{u}_{1}-\frac{1}{H} \int_{-h}^{\alpha \eta} \boldsymbol{u}_{1} \mathrm{~d} z
$$


and the following equality holds:

$$
\int_{-h}^{\alpha \eta} \boldsymbol{V} \mathrm{d} z=0
$$

Concerning the vertical velocity component, since (3.5) contains the factor $\mu^{2}$ before parentheses, it is sufficient to obtain its representation up to terms of order $O\left(\mu^{2}\right)$ only. Taking into account representation (3.11), formula (3.14), and the formula $\boldsymbol{u}_{0}=\boldsymbol{c}+O\left(\mu^{2}\right)$ following from (3.15), we get

$$
w(z)=-D h-(z+h) \nabla \cdot c+O\left(\mu^{2}\right)
$$

where, in contrast with (2.3), we have

$$
D=\frac{\partial}{\partial t}+\boldsymbol{c} \cdot \nabla
$$

Expressions (3.16), (3.19) are used below to obtain the formula for the pressure and for derivation of NLD motion equations.

\subsection{Derivation of NLD motion equations}

We derive the formula expressing the pressure through the variables of the NLD model. To do that, integrate equation (3.5) over the vertical coordinate from $z$ to $\alpha \eta(-h \leqslant z \leqslant \alpha \eta)$ subject to conditions (3.7) and, applying the formula $\boldsymbol{u}=\boldsymbol{c}+O\left(\mu^{2}\right)$ following from (3.16), write down the result in the form of the relation

$$
p(z)=\mu^{2} \int_{z}^{\alpha \eta}\left(D w+w w_{\zeta}+O\left(\mu^{2}\right)\right) \mathrm{d} \zeta-z+\alpha \eta
$$

Let us transform the integrand using formula (3.19):

$$
D w+w w_{z}=-D^{2} h-(z+h) D(\nabla \cdot \boldsymbol{c})+(z+h)(\nabla \cdot \boldsymbol{c})^{2}+O\left(\mu^{2}\right)-R_{2}-(z+h) R_{1}+O\left(\mu^{2}\right) .
$$

Here, in contrast with (2.3), we have

$$
R_{1}=D(\nabla \cdot \boldsymbol{c})-(\nabla \cdot \boldsymbol{c})^{2}, \quad R_{2}=D^{2} h .
$$

Substituting the obtained relation into expression (3.21), we derive the following formula of pressure distribution over the coordinate $z(-h \leqslant z \leqslant \alpha \eta)$ :

$$
p=H-(z+h)-\mu^{2}\left[(H-(z+h)) R_{2}+\left(\frac{H^{2}}{2}-\frac{(z+h)^{2}}{2}\right) R_{1}\right]+O\left(\mu^{4}\right) .
$$

Using relations (3.16), (3.19), and (3.23), derive the motion equations of the NLD model. To do that, integrate equation (3.4) over the water layer thickness and apply condition (3.7). This gives the relation

$$
\int_{-h}^{\alpha \eta}\left(\boldsymbol{u}_{t}+(\boldsymbol{u} \cdot \nabla) \boldsymbol{u}+w \boldsymbol{u}_{z}\right) \mathrm{d} z+\nabla \int_{-h}^{\alpha \eta} p \mathrm{~d} z-\left.p\right|_{z=-h} \nabla h=0 .
$$

Transform it using the representations of $\boldsymbol{u}, w$, and $p$ through the variables $\boldsymbol{c}, H$. In order to calculate the terms with the pressure, we use formula (3.23):

$$
\nabla \int_{-h}^{\alpha \eta} p \mathrm{~d} z-\left.p\right|_{z=-h} \nabla h=\alpha H \nabla \eta-\mu^{2}\left[\nabla\left(\frac{H^{3}}{3} R_{1}+\frac{H^{2}}{2} R_{2}\right)-H \nabla h\left(\frac{H}{2} R_{1}+R_{2}\right)\right]+O\left(\mu^{4}\right) .
$$

Further, applying formulas (3.16), (3.19), we calculate the terms with the vertical velocity component. We have

$$
\int_{-h}^{\alpha \eta} w \boldsymbol{u}_{z} \mathrm{~d} z=-\mu^{2} \int_{-h}^{\alpha \eta}[D h+(z+h)(\nabla \cdot \boldsymbol{c})] \boldsymbol{V}_{z} \mathrm{~d} z+O\left(\mu^{4}\right)=-\left.\mu^{2} D h \boldsymbol{V}\right|_{z=-h} ^{z=\alpha \eta}-\mu^{2}(\nabla \cdot \boldsymbol{c}) \int_{-h}^{\alpha \eta}(z+h) \boldsymbol{V}_{z} \mathrm{~d} z+O\left(\mu^{4}\right) .
$$


The integral in the right-hand side of the obtained relation is calculated by parts, which, due to equality (3.18), gives the formula

$$
\int_{-h}^{\alpha \eta}(z+h) \boldsymbol{V}_{z} \mathrm{~d} z=\left.H \boldsymbol{V}\right|_{z=\alpha \eta}
$$

Taking into account the latter formula, we get

$$
\int_{-h}^{\alpha \eta} w \boldsymbol{u}_{z} \mathrm{~d} z=\left.\mu^{2} D h \boldsymbol{V}\right|_{z=-h}-\left.\mu^{2}(D h+H(\nabla \cdot \boldsymbol{c})) \boldsymbol{V}\right|_{z=\alpha \eta}+O\left(\mu^{4}\right) .
$$

The group of terms of equation (3.24) containing horizontal velocity components is transformed by similar technique including the substitution of expression (3.16), carrying out the differentiation from the integral sign, and application of equality (3.18):

$$
\begin{aligned}
& \int_{-h}^{\alpha \eta}\left[\boldsymbol{u}_{t}\right.+(\boldsymbol{u} \cdot \nabla) \boldsymbol{u}] \mathrm{d} z=\int_{-h}^{\alpha \eta}\left[\boldsymbol{c}_{t}+(\boldsymbol{c} \cdot \nabla) \boldsymbol{c}\right] \mathrm{d} z+\mu^{2} \int_{-h}^{\alpha \eta}\left[\boldsymbol{V}_{t}+(\boldsymbol{c} \cdot \nabla) \boldsymbol{V}+(\boldsymbol{V} \cdot \nabla) \boldsymbol{c}\right] \mathrm{d} z+O\left(\mu^{4}\right) \\
&=H \boldsymbol{c}_{t}+H(\boldsymbol{c} \cdot \nabla) \boldsymbol{c}+\mu^{2}\left[-\left.\alpha \eta_{t} \boldsymbol{V}\right|_{z=\alpha \eta}-\left.h_{t} \boldsymbol{V}\right|_{z=-h}-\left.\alpha(\boldsymbol{c} \cdot \nabla \eta) \boldsymbol{V}\right|_{z=\alpha \eta}-\left.(\boldsymbol{c} \cdot \nabla h) \boldsymbol{V}\right|_{z=-h}\right]+O\left(\mu^{4}\right) .
\end{aligned}
$$

We combine the obtained expression with the right-hand side of (3.26) and transform the terms in $O\left(\mu^{2}\right)$ using continuity equation (3.10) in the form

$$
D H+H(\nabla \cdot \boldsymbol{c})=0
$$

and also the equality $h_{t}+\boldsymbol{c} \cdot \nabla h-D h=0$. As the result, we get the following approximation with the order $O\left(\mu^{4}\right)$ of the acceleration averaged over depth:

$$
\int_{-h}^{\alpha \eta}\left[\boldsymbol{u}_{t}+(\boldsymbol{u} \cdot \nabla) \boldsymbol{u}+w \boldsymbol{u}_{z}\right] \mathrm{d} z=H \boldsymbol{c}_{t}+H(\boldsymbol{c} \cdot \nabla) \boldsymbol{c}+O\left(\mu^{4}\right) .
$$

Returning to equation (3.24) and performing the corresponding substitutions according to the applied transformations and rejecting the terms of order $O\left(\mu^{4}\right)$, we obtain the required motion equation of the NLD model

$$
\boldsymbol{c}_{t}+(\boldsymbol{c} \cdot \nabla) \boldsymbol{c}+\alpha \nabla \eta=\mu^{2}\left[\frac{1}{H} \nabla\left(\frac{H^{3}}{3} R_{1}+\frac{H^{2}}{2} R_{2}\right)-\nabla h\left(\frac{H}{2} R_{1}+R_{2}\right)\right] .
$$

Equations (3.10), (3.29) describe the propagation of long waves with dispersion on a fixed bottom, these equations coincide in form with those derived in [10]. In the case of a fixed bottom, these equations can be reduced to equations from $[17,20]$. Note that in the derivation of NLD equations in $[10,17,20]$ the flow was not assumed potential and the derivation was performed with the use of the velocity potential.

The analysis of derivation of the NLD model proposed above shows that the use of the potentiality condition allows us to obtain an explicit expression for the coefficient $\boldsymbol{u}_{1}$ in the expansion of the velocity $\boldsymbol{u}$ by formula (3.11) and hence to calculate $\boldsymbol{V}$ by formula (3.17). As shown in this paper, such explicit formulas are not needed for the derivation of motion equations in an NLD model, it is only sufficient to have relation (3.18).

Formula (3.23) implies the following formula for calculation of the pressure in the NLD model:

$$
\tilde{p}=H-(z+h)-\mu^{2}\left[(H-(z+h)) R_{2}+\left(\frac{H^{2}}{2}-\frac{(z+h)^{2}}{2}\right) R_{1}\right] .
$$

Thus, in this case, in contrast with the first approximation of the shallow water theory, we have a quadratic dependence of $\widetilde{p}$ on the vertical coordinate. If we pass to dimensionless variables in formula (3.30), then it coincides with the formula for the pressure in the Green-Naghdi model.

If we denote mean pressure over layer thickness (3.30) by $P$ :

$$
P=\frac{1}{H} \int_{-h}^{\alpha \eta} \tilde{p} \mathrm{~d} z=\frac{H}{2}-\mu^{2}\left(\frac{H^{2}}{3} R_{1}+\frac{H}{2} R_{2}\right)
$$


then motion equation (3.29) can be written similar to the momentum variation equation in the gas dynamics:

$$
\boldsymbol{c}_{t}+(\boldsymbol{c} \cdot \nabla) \boldsymbol{c}+\frac{\nabla(P H)}{H}=q \nabla h
$$

in this case,

$$
q=\left.\frac{1}{H} \tilde{p}\right|_{z=-h}=1-\mu^{2}\left(\frac{H}{2} R_{1}+R_{2}\right) .
$$

Multiplying equation (3.10) by $\boldsymbol{c}$ and (3.32) by $H$ and summing up the results, we come to the following notation of the NLD motion equation with a divergent left-hand side:

$$
(H \boldsymbol{c})_{t}+\nabla \cdot(H \boldsymbol{c} \otimes \boldsymbol{c})+\nabla(H P)=q H \nabla h,
$$

where $\boldsymbol{c} \otimes \boldsymbol{c}$ is the tensor product of the vector $\boldsymbol{c}$ by itself. In the case of an even bottom this equation takes a conservative form.

\subsection{Energy balance equation}

Let us analyze three-dimensional equations of hydrodynamics (3.3)-(3.5) in dimensionless variables. It is not difficult to check that the total energy equation corresponding to them coincides in form with equation (1.6), where $E=K+\Pi$, but, in this case, we have $K=\left(u^{2}+v^{2}+\mu^{2} w^{2}\right) / 2$ and $\Pi=z$.

We consider the total energy averaged over the layer thickness. Substituting expansions (3.16), (3.19) into the expression for $E$ instead of the velocity components, we get

$$
\frac{1}{H} \int_{-h}^{\alpha \eta} E \mathrm{~d} z=\frac{1}{2 H} \int_{-h}^{\alpha \eta}\left[\boldsymbol{c} \cdot \boldsymbol{c}+2 \mu^{2} \boldsymbol{c} \cdot \boldsymbol{V}+\mu^{2}(D h+(z+h) \nabla \cdot \boldsymbol{c})^{2}+z\right] \mathrm{d} z+O\left(\mu^{4}\right) .
$$

Performing the integration over $z$, we have

$$
\frac{1}{H} \int_{-h}^{\alpha \eta} E \mathrm{~d} z=\mathcal{E}+O\left(\mu^{4}\right)
$$

where

$$
\varepsilon=\frac{\boldsymbol{c} \cdot \boldsymbol{c}}{2}+\mu^{2}\left(\frac{H^{2}}{6}(\nabla \cdot \boldsymbol{c})^{2}+\frac{H}{2}(\nabla \cdot \boldsymbol{c}) D h+\frac{(D h)^{2}}{2}\right)+\frac{H-2 h}{2} .
$$

Taking into account relation (3.35) between the averaged total energy of the three-dimensional flow and the function $\varepsilon$, the latter can be naturally identified with the total flow energy described with in the NLD model.

We derive the equation for the total energy $\varepsilon$. To do that, multiply vector equation (3.32) by $c$ and take into account the equality $\boldsymbol{c} \cdot(\boldsymbol{c} \cdot \nabla) \boldsymbol{c}=\boldsymbol{c} \cdot \nabla(\boldsymbol{c} \cdot \boldsymbol{c}) / 2$. As the result, we get

$$
D\left(\frac{\boldsymbol{c} \cdot \boldsymbol{c}}{2}\right)+\frac{1}{H} \nabla \cdot(\boldsymbol{c H P})-P \nabla \cdot \boldsymbol{c}-q D h=-q h_{t} .
$$

Using notations (3.22) and formulas (3.27), (3.31), (3.33), we come to the following chain of equalities:

$$
\begin{aligned}
P \nabla \cdot \boldsymbol{c}+q D h= & \frac{H}{2} \nabla \cdot \boldsymbol{c}+D h-\mu^{2}\left[\left(\frac{H^{2}}{3} R_{1}+\frac{H}{2} R_{2}\right) \nabla \cdot \boldsymbol{c}+D h\left(\frac{H}{2} R_{1}+R_{2}\right)\right] \\
= & -\frac{D H}{2}+D h-\mu^{2}\left[\frac{H}{3}(\nabla \cdot \boldsymbol{c})^{2} D H+\frac{H^{2}}{3} D \frac{(\nabla \cdot \boldsymbol{c})^{2}}{2}+\frac{H}{2}(\nabla \cdot \boldsymbol{c}) D^{2} h+\frac{H}{2} D(\nabla \cdot \boldsymbol{c}) D h\right. \\
& \left.+\frac{D h}{2}(\nabla \cdot \boldsymbol{c}) D H+D h D^{2} h\right] \\
= & -D\left(\frac{H-2 h}{2}\right)-\mu^{2}\left[D\left(\frac{H^{2}}{6}(\nabla \cdot \boldsymbol{c})^{2}\right)+D\left(\frac{H}{2}(\nabla \cdot \boldsymbol{c}) D h\right)+D\left(\frac{(D h)^{2}}{2}\right)\right]=-D\left(\varepsilon-\frac{\boldsymbol{c} \cdot \boldsymbol{c}}{2}\right) .
\end{aligned}
$$


Taking into account the latter equality, we can rewrite equation (3.37) in the form

$$
\frac{\partial \mathcal{E}}{\partial t}+\boldsymbol{c} \cdot \nabla \mathcal{E}+\frac{1}{H} \nabla \cdot(\boldsymbol{c} H P)=-q h_{t} .
$$

If we multiply this equation by $H$, continuity equation (3.10) by $\varepsilon$, and sum the results, we obtain the total energy equation in the NLD model having a divergent form of the left-hand side:

$$
\frac{\partial H \varepsilon}{\partial t}+\nabla \cdot(H \boldsymbol{c}(\varepsilon+P))=-q H h_{t} .
$$

This equation can be derived directly from the total energy conservation law in the three-dimensional model. If we transform equation (1.7) to dimensionless form, substitute expansions (3.16), (3.19) and expression for the pressure (3.23) into it, and apply appropriate calculations, then we can get the following relation:

$$
\frac{\partial}{\partial t} \int_{-h}^{\alpha \eta} E \mathrm{~d} z+\nabla \cdot \int_{-h}^{\alpha \eta} \boldsymbol{u}(E+p) \mathrm{d} z+\left.p\right|_{z=-h} h_{t}=\frac{\partial H \mathcal{E}}{\partial t}+\nabla \cdot(H \boldsymbol{c}(\mathcal{E}+P))+q H h_{t}+O\left(\mu^{4}\right)
$$

i.e., equation (3.39) approximates the total energy conservation law for the original hydrodynamic system averaged over depth with the order $O\left(\mu^{4}\right)$. Taking into account (3.40), equation (3.39) can be called the total energy balance equation describing the total energy variation in the NLD model.

If we pass to dimensionless variables, then the obtained law of total energy variation in the NLD model, as well as the total energy formula itself, formally coincides with those obtained for the Green-Naghdi model. This is to be expected because the governing equations of the Green-Naghdi model and complete NLD model from this paper are equivalent (this issue was discussed in [10]).

For $h_{t}=0$, i.e., in the case of a fixed bottom, energy equation (3.39) takes a divergent form and can be reduced to the energy conservation law from [1] by algebraic transformations.

\section{Boussinesq type model with energy balance equation}

Deriving NLD equations (3.10), (3.32), we do not use the assumption on the smallness of wave amplitudes. Assuming a certain relation between the parameters $\alpha$ and $\mu^{2}$, we come to Boussinesq type equations differing from the complete NLD model in a simpler form of dispersion terms.

Based on equations (3.10), (3.32), we obtain approximate NLD equations for waves of small amplitude and show that these equations also admit an energy equation of form (3.39) as a corollary.

Using continuity equation (3.27), write the expression for $R_{1}$ in the following form:

$$
R_{1}=\frac{D(H \nabla \cdot \boldsymbol{c})}{H} .
$$

In this case, relations (3.31), (3.33) imply the equalities

$$
P=\frac{H}{2}-\mu^{2}\left(\frac{H}{3} D(H \nabla \cdot \boldsymbol{c})+\frac{H}{2} D^{2} h\right), \quad q=1-\mu^{2}\left(\frac{1}{2} D(H \nabla \cdot \boldsymbol{c})+D^{2} h\right) .
$$

Taking into account $H=h+\alpha \eta$ and neglecting the terms of orders $O\left(\alpha \mu^{2}\right), O\left(\alpha^{2} \mu^{2}\right)$ in expressions (4.2), we get motion equations (3.32) of the weakly dispersive model with modified functions $P$ and $q$ :

$$
P=\frac{H}{2}-\mu^{2}\left(\frac{h}{3} D(h \nabla \cdot \boldsymbol{c})+\frac{h}{2} D^{2} h\right), \quad q=1-\mu^{2}\left(\frac{1}{2} D(h \nabla \cdot \boldsymbol{c})+D^{2} h\right)
$$

and the following expression for the total energy:

$$
\mathcal{E}=\frac{(\boldsymbol{c} \cdot \boldsymbol{c})}{2}+\frac{H-2 h}{2}+\mu^{2}\left(\frac{h^{2}}{6}(\nabla \cdot \boldsymbol{c})^{2}+\frac{h}{2}(\nabla \cdot \boldsymbol{c}) D h+\frac{(D h)^{2}}{2}\right) .
$$


The equation describing the variation of energy (4.4) is derived by the analogy with equation (3.38), i.e., we get equation (3.37) first and then calculate the last summand from its left-hand side

$$
P \nabla \cdot \boldsymbol{c}+q D h=-D\left(\frac{H-2 h}{2}\right)-\mu^{2}\left[D\left(\frac{h^{2}}{6}(\nabla \cdot \boldsymbol{c})^{2}\right)+D\left(\frac{h}{2}(\nabla \cdot \boldsymbol{c}) D h\right)+D\left(\frac{(D h)^{2}}{2}\right)\right]=-D\left(\varepsilon-\frac{(\boldsymbol{c} \cdot \boldsymbol{c})}{2}\right) .
$$

By the analogy with the case considered above, we come to the form of energy equation with a divergent left-hand side coinciding with equation (3.39) for the complete NLD model.

Thus, in equations of weakly dispersive model (3.10), (3.32) the mean pressure and relative pressure are calculated by formulas (4.3) and total energy (4.4) satisfies equations (3.38) and (3.39). We should note another important property of this model, it is invariant under a Galilean transformation, which can be easily verified by direct checking.

\section{Energy balance in a Boussinesq type model for weakly deformable bottom}

In many practical situations, the translation of the bottom surface in time is small compared to the local depth of water area in a period typical for propagation of waves of tsunami type. Thus, we can assume that

$$
h(x, y, t)=h_{1}(x, y)+\varepsilon h_{2}(x, y, t),
$$

where $\varepsilon$ is small [6]. The study of different regimes of long-wave generation by a movable bottom shows that the wave amplitudes on the water surface are proportional to the shift of bottom surface and hence for a certain class of problems we can assume that

$$
O(\alpha)=O(\varepsilon)=O\left(\mu^{2}\right)
$$

(see, e.g. $[6,7])$. In this case, we have

$$
h=h_{1}(x, y)+O(\alpha), \quad H(x, y, t)=h_{1}(x, y)+O(\alpha) .
$$

Assumption (5.2) allows us to obtain a weakly dispersive model of surface wave propagation above a weakly deformable bottom. This model admits an energy equation similar to (3.39). The derivation of the model is based on the use of the original NLD equations in form (3.10), (3.32) where the expressions for $P$ and $q$ have form (4.2) due to (4.1).

Using approximations (5.3) and neglecting the terms of the order $O\left(\alpha \mu^{2}\right)$ and higher we get motion equations (3.32) with simplified functions $P$ and $q$. In this case, the parentheses for $\mu^{2}$ contain only the stationary part of the bottom surface

$$
P=\frac{H}{2}-\mu^{2}\left(\frac{h_{1}}{3} D\left(h_{1} \nabla \cdot \boldsymbol{c}\right)+\frac{h_{1}}{2} D^{2} h_{1}\right), \quad q=1-\mu^{2}\left(\frac{1}{2} D\left(h_{1} \nabla \cdot \boldsymbol{c}\right)+D^{2} h_{1}\right)
$$

and the right-hand side takes the form

$$
\nabla h+(q-1) \nabla h_{1} .
$$

Under the same conditions, we have the following expression for total energy (3.36):

$$
\varepsilon=\frac{(\boldsymbol{c} \cdot \boldsymbol{c})}{2}+\frac{H-2 h}{2}+\mu^{2}\left(\frac{h_{1}^{2}}{6}(\nabla \cdot \boldsymbol{c})^{2}+\frac{h_{1}}{2}(\nabla \cdot \boldsymbol{c}) D h_{1}+\frac{\left(D h_{1}\right)^{2}}{2}\right) .
$$

In order to derive equations describing the variation of total energy (5.5), all the calculations are performed exactly without removing any terms. This allows us to consider the energy equations as an exact corollary of the motion and continuity equations. 
Proceeding to derivation, multiply vector equation (3.32) by $\boldsymbol{c}$ and write it by the analogy with (3.37) in the form

$$
D\left(\frac{\boldsymbol{c} \cdot \boldsymbol{c}}{2}\right)+\frac{1}{H} \nabla \cdot(\boldsymbol{c} H P)-P \nabla \cdot \boldsymbol{c}-D h-(q-1) D h_{1}=-\left(h-h_{1}\right)_{t} .
$$

Let us transform this equation. First, by the analogy with previous sections, we apply a chain of transformations which, as in the cases considered above, leads to the relation

$$
P \nabla \cdot \boldsymbol{c}+D h+(q-1) D h_{1}=-D\left(\varepsilon-\frac{(\boldsymbol{c} \cdot \boldsymbol{c})}{2}\right)
$$

where the energy $\varepsilon$ has form (5.5). Taking into account the obtained relations, we get the equation

$$
\frac{\partial \mathcal{E}}{\partial t}+\boldsymbol{c} \cdot \nabla \mathcal{E}+\frac{1}{H} \nabla \cdot(\boldsymbol{c H P})=-h_{t}
$$

Multiplying this equation by $H$, continuity equation (3.10) by $\mathcal{E}$, and summing the results, we get the total energy equation of the NLD model for weakly dispersive flows over a weakly deformable bottom having a divergent form of the left-hand side:

$$
\frac{\partial H \varepsilon}{\partial t}+\nabla \cdot(H c(\varepsilon+P))=-H h_{t} .
$$

Note in conclusion that the term $D h_{1}$ can be replaced by $c \cdot \nabla h_{1}$ in formulas (5.4), (5.5).

\section{Conclusion}

A derivation of nonlinear-dispersion shallow water equations not using the potentiality of the original threedimensional flow is presented in the paper. New models of Boussinesq type are obtained for flows above a fixed or slowly varying uneven bottom are obtained. All obtained models have the same structure of equations, which allows one to use a unified approach for construction of numerical algorithms.

Total energy variation laws are derived for complete NLD equations and for two weakly dispersive models in the case of movable bottom surface. These laws are corollaries of obtained NLD equations and have an important value for verification of numerical algorithms. The important circumstance is the consistency of energy balance equations of the approximate and three-dimensional hydrodynamic models consisting in the fact that each derived energy balance equation can be obtained from the energy conservation law of Euler equations taking into account the order of approximation with which this model approximates the threedimensional flow model.

We emphasize the main features of the weakly dispersive models of Boussinesq type obtained here. These are the presence of physically meaningful laws of momentum and energy variation and the invariance relative to Galilean transformations. We can say that these simplified models keep very important physical properties of complete NLD models, which, in their turn, inherit these properties from the three-dimensional models of hydrodynamics. This fact distinguishes the models obtained in this paper from other well-known models of Boussinesq type (see, e.g., $[6,16])$.

Funding: The work was supported by the Russian Foundation for Basic Research (12-01-00721-a) and also by the program 'Leading scientific schools of the Russian Federation' (NSh-6293.2012.9).

\section{References}

[1] S. V. Bazenkov, N. N. Morozov, and O. P. Pogutse, Dispersive effects in two-dimensional hydrodynamics. Doklady Akad. Nauk SSSR 293 (1987), No. 4, 818-822 (in Russian).

[2] J. L. Bona, T. Colin, and D. Lannes, Long wave approximations for water waves. Arch. Rational Mech. Anal. 178 (2005), $373-$ 410. 
[3] J. D. Carter and R. Cienfuegos, The kinematics and stability of solitary and cnoidal wave solutions of the Serre equations. Eur. J. Mech. B/Fluids 30 (2011), 259-268.

[4] D. Clamond and D. Dutykh, Practical use of variational principles for modelling water waves. Physica D 241 (2012), No. 1, 25-36.

[5] F. Dias and P. Milewski, On the fully-nonlinear shallow-water generalized Serre equations. Physics Letters A 374 (2010), No. 8, 1049-1053.

[6] A. A. Dorfman and G. I. Yagovdik, The equations of approximate nonlinear-dispersive theory of long gravitational waves caused by a moving bottom and propagating in a basin of variable depth. Chisl. Metody Mekhan. Sploshn. Sredy 8 (1977), No. 1, 36-48 (in Russian).

[7] D. Dutykh and F. Dias, Energy of tsunami waves generated by bottom motion. Proc. R. Soc. A. 465 (2009), 725-744.

[8] R. C. Ertekin, W. C. Webster, and J. V. Wehausen, Waves caused by a moving disturbance in a shallow channel of finite width. J. Fluid Mech. 169 (1986), 275-292.

[9] Z. I. Fedotova and E. D. Karepova, Variational principle for approximate models of wave hydrodynamics. Russ. J. Numer. Anal. Math. Modelling 11 (1996), No. 3, 183-204.

[10] Z. I. Fedotova and G. S. Khakimzyanov, Shallow water equations on a movable bottom. Russ. J. Numer. Anal. Math. Modelling 24 (2009), No. 1, 31-41.

[11] Z. I. Fedotova and G. S. Khakimzyanov, Full nonlinear dispersion model of shallow water equations on a rotating sphere. J. Appl. Mech. and Tech. Phys. 52 (2011), No. 6, 865-876.

[12] A. E. Green and P. M. Naghdi, A derivation of equations for wave propagation in water of variable depth. J. Fluid Mech. 78 (1976), No. 2, 237-246.

[13] D. Lannes and P. Bonneton, Derivation of asymptotic two-dimensional time-dependent equations for surface water wave propagation. Phys. Fluids 21 (2009), 16601-16609.

[14] Y. A. Li, Hamiltonian structure and linear stability of solitary waves of the Green-Naghdi equations. J. Nonlin. Math. Phys. 9 (2002), No. 1, 99-105.

[15] L. V. Ovsyannikov, N. I. Makarenko, V. I. Nalimov, et al., Nonlinear Problems in the Theory of Surface and Internal Waves. Nauka, Novosibirsk, 1985 (in Russian).

[16] D. H. Peregrine, Long waves on a beach. J. Fluid. Mech. 27 (1967), No. 4, 815-827.

[17] N. E. Voltzinger, K. A. Klevanny, and E. N. Pelinovsky, Long-Wave Dynamics of the Coastal Zone. Gidrometeoizdat, Leningrad, 1989 (in Russian).

[18] N. E. Voltsinger and R. V. Pyaskovskii, The Theory of Shallow Water, Gidrometeoizdat, Leningrad, 1977 (in Russian).

[19] G. B. Whitham, Linear and Nonlinear Waves. John Wiley\&Sons, Inc., New York, 1974.

[20] M. I. Zheleznyak and E. N. Pelinovsky, Physico-mathematical models of the tsunami climbing a beach. In: Tsunami Climbing a Beach (Ed. E. N. Pelinovsky). IAP Akad. Sci. USSR, Gorky, 1985, pp. 8-33 (in Russian).

Received June 10, 2013; accepted February 25, 2014. 